\title{
A142 INTRAVENOUS IMMUNOGLOBULIN INHIBITS TOLL-LIKE RECEPTOR 9-INDUCED ACTIVATION OF AUTO-REACTIVE B LYMPHOCYTES
}

Sophie Hillion, Jacques-Olivier Pers, Seité Jean-François, Divi Cornec, Alain Saraux, Pierre Youinou Brest University Medical School, Brest, France

\subsection{6/ard.2010.149005.9}

Background Inappropriate toll-like receptor (TLR) 9 signaling contributes to the activation of anti-dsDNA antibody-producing B cells in systemic lupus erythematosus (SLE). IVIg, which modulates $B$ cell response through a sialic acid-CD22 lectin interaction (Hillion, Blood 2010), might also acts by repressing the TLR9 signal transduction.

Material and methods Blood and tonsil mononuclear cell suspensions were enriched in B cells. TLR4, 7-8 and 9 were engaged using LPS, CL097 and CpG oligonucleotides, respectively. The SHP-1 phosphatase was inhibited by methyl 3-4 dephostatin, and the MyD 88 adaptor of TLRs by a specific blocking peptide.

Results IVIg prevented the in vitro activation of TLR7 and TLR9, as shown by CD25, CD40 and class II-MHC downregulation. For this to occur, IVIg has to be continuously present in the culture. Furthermore, IVIg suppresses the production of the proinflammatory IL- 6 as well as the immunostimulatory sequence-induced production of the anti-inflammatory IL-10. There was also an IVIg-mediated reduction of CXCR4 and CXCR5, two chemokines receptors dependent on engagement of the TLRs by their agonists. Finally, there appeared that the SHP-1 phosphatase was recruited by IVIg, as a feature of the B cell modulation.

Conclusion Taken together, our data suggest that IVIg could repress the TLR9-induced activation of B cells in autoimmunity, along with the expression of pro-inflammatory cytokines. This opens up a new therapeutic avenue in SLE and related diseases. 